\title{
Multipartite generalisation of the Schmidt decomposition
}

\author{
H. A. Carteret $^{1}$, A. Higuchi ${ }^{2}$ and A. Sudbery ${ }^{3}$ \\ Dept. of Mathematics, University of York, Heslington, York, YO10 5DD, U.K. \\ email: ${ }^{1}$ hac100@york.ac.uk, ${ }^{2}$ ah28@york.ac.uk, ${ }^{3}$ as2@york.ac.uk
}

27 June 2000, revised 15 November 2000

\begin{abstract}
We find a canonical form for pure states of a general multipartite system, in which the constraints on the coordinates (with respect to a factorisable orthonormal basis) are simply that certain ones vanish and certain others are real. For identical particles they are invariant under permutations of the particles. As an application, we find the dimension of the generic local equivalence class.
\end{abstract}

\section{Introduction}

Recently considerable attention [1, 2, 3, 4, 6, 7, 8, 9, 11, 12, 13, 5] has been devoted to the problem of describing the equivalence classes of states of a composite quantum system, where two states are regarded as equivalent if they are related by a unitary transformation which factorises into separate transformations on the component parts (a local unitary transformation). One approach to this problem is to specify a canonical form for states under local unitary transformations. For pure states of two-part systems, such a canonical form is given by the Schmidt decomposition

$$
|\Psi\rangle=\sum_{i} \alpha_{i}\left|\phi_{i}\right\rangle\left|\psi_{i}\right\rangle
$$

where the $\left|\phi_{i}\right\rangle$ are a set of orthogonal states of the first subsystem, the $\left|\psi_{i}\right\rangle$ are a set of orthogonal states of the second subsystem, and the $\alpha_{i}$ are positive real 
numbers. The state $|\Psi\rangle$ is thus expanded in terms of a factorisable basis of two-part states so that the number of non-zero coefficients is minimal. Acín et al [1]] have shown that there is an expansion of states of three qubits which has a similar property. In this note we will demonstrate such a decomposition for pure states of an $n$-part system, where the dimensions of the individual state spaces are finite but otherwise arbitrary. Then we use this to find the dimension of the generic local equivalence class. We will also comment on the relation of this decomposition to other proposed canonical forms.

\section{The generalised Schmidt decomposition}

We first state and prove the generalisation of the Schmidt decomposition for a multipartite state in which all the individual state spaces have the same dimension, since this is considerably simpler than the general case:

Theorem 1. Let $|\Psi\rangle$ be a state vector in an $n$-fold tensor product space $\mathcal{S}_{1} \otimes \cdots \otimes \mathcal{S}_{n}$ where $\operatorname{dim} \mathcal{S}_{1}=\cdots=\operatorname{dim} \mathcal{S}_{n}=d \geq 2$ and $n \geq 3$. Then for $r=1, \ldots, n$ there is a basis $\left\{\left|\psi_{i}^{(r)}\right\rangle: i=1, \ldots, d\right\}$ of $\mathcal{S}_{r}$ such that in the expansion

$$
|\Psi\rangle=\sum_{i_{1} \cdots i_{n}} c_{i_{1} \cdots i_{n}}\left|\psi_{i_{1}}^{(1)}\right\rangle \cdots\left|\psi_{i_{n}}^{(n)}\right\rangle
$$

the coefficients $c_{i_{1} \cdots i_{n}}$ have the following properties:

1. $c_{j i i \cdots i}=c_{i j i \cdots i}=\cdots=c_{i i \cdots i j}=0$ if $1 \leq i<j \leq d$;

2. $c_{i_{1} \cdots i_{n}}$ is real and non-negative if at most one of the $i_{r}$ differs from $d$;

3. $\left|c_{i i \ldots i}\right| \geq\left|c_{j_{1} \ldots j_{n}}\right|$ if $i \leq j_{r}, r=1, \ldots, n$.

Proof. Consider the real-valued function $\mid\left\langle\Psi\left|\left(\left|\phi^{(1)}\right\rangle \cdots\left|\phi^{(n)}\right\rangle\right)\right|^{2}\right.$ defined for unit vectors $\left|\phi^{(r)}\right\rangle$ lying in the unit sphere $S^{2 d-1}$ in $\mathcal{H}_{r}$. As $\left(\left|\phi^{(1)}\right\rangle, \ldots,\left|\phi^{(n)}\right\rangle\right)$ varies over the compact space $S^{2 d-1} \times \cdots \times S^{2 d-1}$, this function attains a maximum at some point $\left(\left|\psi_{1}^{(1)}\right\rangle, \ldots,\left|\psi_{1}^{(n)}\right\rangle\right)$. Let $\left\{\left|\psi_{i}^{(r)}\right\rangle: i=1, \ldots, d\right\}$ be any orthonormal basis of $\mathcal{H}_{r}$ containing $\left|\psi_{1}^{(r)}\right\rangle$, and expand $|\Psi\rangle$ as in the statement of the theorem. Since $\left|\left\langle\Psi\left|\left(\left|\psi_{1}^{(1)}\right\rangle \cdots\left|\phi^{(r)}\right\rangle \cdots\left|\psi_{1}^{(n)}\right\rangle\right)\right|^{2}\right.\right.$ is stationary at $\left|\phi^{(r)}\right\rangle=\left|\psi_{1}^{(r)}\right\rangle$ for variations of $\left|\phi^{(r)}\right\rangle$ on the unit sphere,

$$
c_{1 \ldots 1 j 1 \ldots 1}=\left\langle\Psi \left|\left(\left|\psi_{1}^{(1)}\right\rangle \cdots\left|\psi_{1}^{(r-1)}\right\rangle\left|\psi_{j}^{(r)}\right\rangle\left|\psi_{1}^{(r+1)}\right\rangle \cdots\left|\psi_{1}^{(n)}\right\rangle\right)=0 \quad \text { for } \quad j>1 .\right.\right.
$$


Next, find the maximum of $\mid\left\langle\Psi\left|\left(\left|\phi^{(1)}\right\rangle \cdots\left|\phi^{(n)}\right\rangle\right)\right|^{2}\right.$ as $\left.\mid \phi^{(1)}\right\rangle, \ldots,\left|\phi^{(n)}\right\rangle$ vary over unit vectors orthogonal to $\left|\psi_{1}^{(1)}\right\rangle, \ldots,\left|\psi_{1}^{(n)}\right\rangle$ respectively. Suppose the maximum occurs at $\left(\left|\psi_{2}^{(1)}\right\rangle, \ldots,\left|\psi_{2}^{(n)}\right\rangle\right)$. Then, as before, in any expansion of $|\Psi\rangle$ in terms of orthonormal bases of the $\mathcal{H}_{r}$ containing $\left|\psi_{1}^{(r)}\right\rangle$ and $\left|\psi_{2}^{(r)}\right\rangle, \quad(r=1, \ldots, n)$, the coefficients will satisfy

$$
c_{2 \ldots 2 j 2 \ldots 2}=0 \text { for } j>2 \text {. }
$$

Continuing in this way, we define the basis vectors $\left|\psi_{i}^{(1)}\right\rangle, \ldots,\left|\psi_{i}^{(n)}\right\rangle$ for $i=$ $1, \ldots, d-1$. Then the last basis vector $\left|\psi_{d}^{(1)}\right\rangle$ of $\mathcal{H}_{1}$ is determined up to phase.

The reality conditions can be imposed as follows. Choose the phase of the basis element $\left|\psi_{i}^{(r)}\right\rangle, \quad i=1, \ldots, d-1$ so that

$$
\arg \left(c_{d \ldots d i d \ldots d}\right)=0
$$

where the index $i$ occurs in the $r$ th place, and then fix $c_{d \ldots d}$ by choosing the phase of $\left|\psi_{d}^{(1)}\right\rangle$.

The general form of the theorem is rather more complicated than the above; to state it, we need to define the following sets of $n$-tuples.

Let $\left(I_{1}, \ldots, I_{N}\right)$ be the set of $(n-1)$-tuples $\left(i_{1}, \ldots, i_{n-1}\right)$ with $1 \leq i_{r} \leq d_{r}$, excluding those of the form $(i, \ldots, i)$ with $1 \leq i<d_{1}$ and those of the form $\left(d_{1}, \ldots, d_{r}, i, \ldots, i\right)$ with $d_{r} \leq i<d_{r+1}$ and $1 \leq r \leq n-2$. We order these $(n-1)$-tuples in lexicographical order, so that $I_{N}=\left(d_{1}, \ldots, d_{n-1}\right)$. Let $D=d_{1} \cdots d_{n-1}$, so that $N=D-d_{n-1}+1$. We define $A$ to be the set of $n$-tuples $\left(i_{1}, \ldots, i_{n}\right)$ with $\left(i_{1}, \ldots, i_{n-1}\right)=I_{k}$ and $i_{n}=d_{n-1}+l$ where $1 \leq k \leq \min \left(N, d_{n}-d_{n-1}\right)$ and $k \leq l \leq d_{n}-d_{n-1}$.

We also define the following sets of $n$-tuples:

$$
\begin{aligned}
B_{1}= & \left\{\left(d_{1}, \ldots, d_{r-1}, j, d_{r+1}, \ldots, d_{n-1}, d_{n-1}\right): 1 \leq j<d_{r}, 1 \leq r \leq n-2\right\}, \\
B_{2}= & \left\{\left(d_{1}, \ldots, d_{n-2}, j, d_{n-1}\right): 1 \leq j<d_{n-2}\right\}, \\
B_{3}= & \left\{\left(d_{1}, \ldots, d_{n-2}, j, 1\right): d_{n-2} \leq j \leq d_{n-1}\right\} \\
B_{4}= & \left\{(i, i, \ldots, i): 2 \leq i \leq d_{1}\right\} \\
& \cup\left\{\left(d_{1}, \ldots, d_{r}, i, \ldots, i\right): 1 \leq r<n-1, d_{r}<i \leq d_{r+1}\right\} \\
& \cup\left\{\left(d_{1}, \ldots, d_{n-1}, i\right): d_{n-1}<i \leq \min \left(D, d_{n}\right)\right\}
\end{aligned}
$$

Then $A, B_{1}, \ldots, B_{4}$ are all disjoint. In terms of these sets, the general Schmidt decomposition can be stated as follows: 
Theorem 2. Let $|\Psi\rangle$ be a state vector in an $n$-fold tensor product space $\mathcal{S}_{1} \otimes \cdots \otimes \mathcal{S}_{n}$ where $\operatorname{dim}_{r}=d_{r}$, with $2 \leq d_{1} \leq \cdots \leq d_{n}$, and $n \geq 3$. Then for $r=1, \ldots, n$ there is a basis $\left\{\left|\psi_{i}^{(r)}\right\rangle: i=1, \ldots, d_{r}\right\}$ of $\mathcal{S}_{r}$ such that in the expansion

$$
|\Psi\rangle=\sum_{i_{1} \cdots i_{n}} c_{i_{1} \cdots i_{n}}\left|\psi_{i_{1}}^{(1)}\right\rangle \cdots\left|\psi_{i_{n}}^{(n)}\right\rangle
$$

the coefficients $c_{i_{1} \cdots i_{n}}$ have the following properties:

1. $c_{i i \cdots i j i i \cdots i}=0$ if $1 \leq i<d_{1}$ and $i<j$.

2. $c_{d_{1} \cdots d_{r} i i \cdots i j i i \cdots i}=0$ if $d_{r} \leq i<d_{r+1}$ and $i<j, 1 \leq r \leq n-2$.

3. $c_{I}=0$ for every $n$-tuple index $I$ in the set $A$.

4. The coefficients with indices in the sets $B_{i}(i=1, \ldots, 4)$ are real and non-negative.

5. For $i=1, \ldots, d_{n-1}$, define

$$
R_{i}=\left|c_{d_{1} \cdots d_{r} i \cdots i}\right|
$$

where $r$ is such that $d_{r}<i \leq d_{r+1}$. Then

$$
R_{1} \geq \cdots \geq R_{d_{n-1}}
$$

Proof. Consider the real-valued function $\mid\left\langle\Psi\left|\left(\left|\phi^{(1)}\right\rangle \cdots\left|\phi^{(n)}\right\rangle\right)\right|^{2}\right.$ defined for unit vectors $\left|\phi^{(r)}\right\rangle$ lying in the unit sphere $S^{2 d_{r}-1}$ in $\mathcal{S}_{r}$. As $\left(\left|\phi^{(1)}\right\rangle, \ldots,\left|\phi^{(n)}\right\rangle\right)$ varies over the compact space $S^{2 d_{1}-1} \times \cdots \times S^{2 d_{n}-1}$, this function attains a maximum at some point $\left(\left|\psi_{1}^{(1)}\right\rangle, \ldots,\left|\psi_{1}^{(n)}\right\rangle\right)$. Let $\left\{\left|\psi_{i}^{(r)}\right\rangle: i=1, \ldots, d_{r}\right\}$ be any orthonormal basis of $\mathcal{S}_{r}$ containing $\left|\psi_{1}^{(r)}\right\rangle$, and expand $|\Psi\rangle$ as in the statement of the theorem. Since $\left|\left\langle\Psi\left|\left(\left|\psi_{1}^{(1)}\right\rangle \cdots\left|\phi^{(r)}\right\rangle \cdots\left|\psi_{1}^{(n)}\right\rangle\right)\right|^{2}\right.\right.$ is stationary at $\left|\phi^{(r)}\right\rangle=\left|\psi_{1}^{(r)}\right\rangle$ for variations of $\left|\phi^{(r)}\right\rangle$ on the unit sphere,

$$
c_{1 \cdots 1 j 1 \cdots 1}=\left\langle\Psi \left|\left(\left|\psi_{1}^{(1)}\right\rangle \cdots\left|\psi_{1}^{(r-1)}\right\rangle\left|\psi_{j}^{(r)}\right\rangle\left|\psi_{1}^{(r+1)}\right\rangle \cdots\left|\psi_{1}^{(n)}\right\rangle\right)=0 \quad \text { for } j>1 .\right.\right.
$$

Next, find the maximum of $\mid\left\langle\Psi\left|\left(\left|\phi^{(1)}\right\rangle \cdots\left|\phi^{(n)}\right\rangle\right)\right|^{2}\right.$ as $\left.\mid \phi^{(1)}\right\rangle, \ldots,\left|\phi^{(n)}\right\rangle$ vary over unit vectors orthogonal to $\left|\psi_{1}^{(1)}\right\rangle, \ldots,\left|\psi_{1}^{(n)}\right\rangle$ respectively. Suppose the maximum occurs at $\left(\left|\psi_{2}^{(1)}\right\rangle, \ldots,\left|\psi_{2}^{(n)}\right\rangle\right)$. Then, as before, in any expansion of $|\Psi\rangle$ in terms of orthonormal bases of the $\mathcal{S}_{r}$ containing $\left|\psi_{1}^{(r)}\right\rangle$ and $\left|\psi_{2}^{(r)}\right\rangle(r=1, \ldots, n)$, the coefficients will satisfy

$$
c_{2 \cdots 2 j 2 \cdots 2}=0 \quad \text { for } \quad j>2 .
$$


Continuing in this way, we define the basis vectors $\left|\psi_{i}^{(1)}\right\rangle, \ldots,\left|\psi_{i}^{(n)}\right\rangle$ for $i=$ $1, \ldots, d_{1}-1$. Then the last basis vector $\left|\psi_{d_{1}}^{(1)}\right\rangle$ of $\mathcal{S}_{1}$ is determined up to phase. Next, if the dimensions $d_{i}$ are not all equal, we maximise $\left|\left\langle\Psi\left|\left(\left|\psi_{d_{1}}^{(1)}\right\rangle\left|\phi^{(2)}\right\rangle \cdots\left|\phi^{(n)}\right\rangle\right)\right|^{2}\right.\right.$ as $\left|\phi^{(2)}\right\rangle, \ldots,\left|\phi^{(n)}\right\rangle$ vary orthogonally to the basis vectors already determined, to find basis vectors $\left|\psi_{d_{1}}^{(2)}\right\rangle, \ldots,\left|\psi_{d_{1}}^{(n)}\right\rangle$; then we find $\left|\psi_{i}^{(2)}\right\rangle, \ldots,\left|\psi_{i}^{(n)}\right\rangle$ for $i=$ $d_{1}+1, \ldots, d_{2}-1$, and hence $\left|\psi_{d_{2}}^{(2)}\right\rangle$; then $\left|\psi_{i}^{(3)}\right\rangle, \ldots,\left|\psi_{i}^{(n)}\right\rangle$ for $i=d_{2}, \ldots, d_{3}-1$; and so on until we have $\left|\psi_{i}^{(n-1)}\right\rangle,\left|\psi_{i}^{(n)}\right\rangle$ for $i=d_{n-2}, \ldots, d_{n-1}-1$. The maximisation at each step implies that the coefficients satisfy (2).

Now, to fix the last $d_{n}-d_{n-1}+1$ basis elements of $\mathcal{S}_{n}$, we choose a set $I_{1}=\left(i_{1}, \ldots, i_{n-1}\right)$ of $n-1$ indices which is not of the form $(i, \ldots, i)$ with $1 \leq$ $i<d_{1}$ or $\left(d_{1}, d_{2}, \ldots, d_{r}, i, i, \ldots, i\right)$ with $d_{r} \leq i<d_{r+1}$ (so that $c_{i_{1} \cdots i_{n-1} j}$ has not yet been set to zero for any $j$ ), and maximise $\left|\left\langle\Psi\left|\left(\left|\psi_{i_{1}}^{(1)}\right\rangle \cdots\left|\psi_{i_{n-1}}^{(n-1)}\right\rangle\left|\phi^{(n)}\right\rangle\right)\right|^{2}\right.\right.$ with respect to vectors $\left|\phi^{(n)}\right\rangle$ orthogonal to $\left|\psi_{1}^{(n)}\right\rangle, \ldots,\left|\psi_{d_{n-1}-1}^{(n)}\right\rangle$, thus finding $\left|\psi_{d_{n-1}}^{(n)}\right\rangle$; then, choosing a different index set $I_{2}=\left(j_{1}, \ldots, j_{n-1}\right)$, maximise $\left|\left\langle\Psi\left|\left(\left|\psi_{j_{1}}^{(1)}\right\rangle \cdots\left|\psi_{j_{n-1}}^{(n-1)}\right\rangle\left|\phi^{(n)}\right\rangle\right)\right|^{2}\right.\right.$ with respect to vectors $\left.\mid \phi^{(n)}\right\rangle$ orthogonal to $\left|\psi_{1}^{(n)}\right\rangle, \ldots,\left|\psi_{d_{n-1}}^{(n)}\right\rangle$; and so on until we have either exhausted the possible index sets $I_{1}, I_{2}, \ldots, I_{N}$ or run out of space in which to vary the vector $\left|\phi^{(n)}\right\rangle$. The coefficients will then satisfy (3).

The reality conditions (4) can be imposed as follows. (By using a basis vector $|\psi\rangle$ to fix a coefficient $c$ we mean changing the phase of $|\psi\rangle$ to make $c$ real and non-negative.) First use $\left|\psi_{1}^{(n)}\right\rangle$ to fix $c_{d_{1} \cdots d_{n-1} 1}$; then use $\left|\psi_{d_{n-1}}^{(n)}\right\rangle$ to fix $c_{d_{1} d_{2} \cdots d_{n-1} d_{n-1}}$; then use $\left|\psi_{j}^{(r)}\right\rangle\left(r=1, \ldots, n-2 ; j=1, \ldots, d_{r}-1\right)$ to fix the coefficients in the set $B_{1}$; then use $\left|\psi_{j}^{(n-1)}\right\rangle\left(j=1, \ldots, d_{n-1}-1\right)$ to fix the coefficients in $B_{2}$ and $B_{3}$; and finally use $\left|\psi_{j}^{(n)}\right\rangle\left(j=2, \ldots, \min \left(d_{n}, D\right)\right.$ excluding $j=d_{n-1}$ ) to fix the remaining coefficients in $B_{4}$.

This result can be expressed in terms of active transformations, with respect to fixed orthonormal bases $\left\{\left|\theta_{i}^{(r)}\right\rangle\right\}$ of the state spaces $\mathcal{S}_{r}$, as follows. Two states $\left|\Psi_{1}\right\rangle,\left|\Psi_{2}\right\rangle$ in $\mathcal{S}_{1} \otimes \cdots \otimes \mathcal{S}_{n}$ are said to be locally equivalent if

$$
\left|\Psi_{1}\right\rangle=\left(U_{1} \otimes \cdots \otimes U_{n}\right)\left|\Psi_{2}\right\rangle
$$

where $U_{r}$ is a unitary transformation acting on $\mathcal{S}_{r}$. Then we have

Theorem 3. For $n \geq 3$, any state $|\Psi\rangle \in \mathcal{S}_{1} \otimes \cdots \otimes \mathcal{S}_{n}$ is locally equivalent to a state $\sum c_{i_{1} \ldots i_{n}}\left|\theta_{i_{1}}^{(1)}\right\rangle \cdots\left|\theta_{i_{n}}^{(n)}\right\rangle$ where the coefficients $c_{i_{1} \ldots i_{n}}$ have the properties 1-5 stated in Theorem 2. 
As an example, we note the canonical form for a state of three qubits,

$$
a|000\rangle+b|011\rangle+c|101\rangle+d|110\rangle+e|111\rangle
$$

with $b, c, d, e$ real. Acín et al [1] have investigated tripartite states using different canonical forms which, like the canonical form proposed here, have five non-zero coefficients of which four are real.

We note that for a state expressed in terms of a fixed orthonormal basis $\left\{\left|\theta_{i}^{(r)}\right\rangle\right\}$ as

$$
|\Psi\rangle=\sum t_{i_{1} \cdots i_{n}}\left|\theta_{i_{1}}^{(1)}\right\rangle \cdots\left|\theta_{i_{n}}^{(n)}\right\rangle
$$

the coefficients of the basis elements $\left|\psi_{1}^{(r)}\right\rangle=\sum u_{i_{r}}^{(r)}\left|\theta_{i_{r}}^{(r)}\right\rangle$ defined in the first step of the above proof are the solutions of the generalised (nonlinear) "singular value" equations

$$
\sum_{i_{1} \cdots i_{r-1}, i_{r+1} \cdots i_{n}} \overline{t_{i_{1} \cdots i_{n}}} u_{i_{1}}^{(1)} \cdots u_{i_{r-1}}^{(r-1)} u_{i_{r+1}}^{(r+1)} \cdots u_{i_{n}}^{(n)}=\lambda \overline{u_{i_{r}}^{(r)}}
$$

where the Lagrange multiplier $\lambda$ is such that $|\lambda|^{2}$ is the maximal value of $\mid\left\langle\Psi\left|\left(\left|\phi^{(1)}\right\rangle \cdots\left|\phi^{(n)}\right\rangle\right)\right|^{2}\right.$.

Let us now examine the dimension of the set of canonical forms and deduce the dimension of the generic local equivalence class. First consider the case where all the individual state spaces have equal dimension $d$. The number of zero coefficients in the canonical form, determined by condition 1 of Theorem 1, is $\frac{1}{2} n d(d-1)$ (one for each pair $(i, j)$ with $i<j$ and for each position of $j$ ). The number of phases removed by condition 2 is $n(d-1)+1$. Hence the number of real parameters in the canonical form of Theorem 1 is

$$
2 d^{n}-n d(d-1)-n(d-1)-1=2 d^{n}-n\left(d^{2}-1\right)-1 .
$$

We have not proved that states with different canonical forms are not locally equivalent; it is conceivable that the number of parameters could be reduced still further by local transformations. However, the difference between the number of parameters in the final canonical form and the dimension of the pure state space must be the dimension of the generic equivalence class, which is therefore at least $n\left(d^{2}-1\right)+1$. But this is the dimension of the group of local unitary transformations, which can be identified with $S U(d)^{n} \times U(1)$ if we collect together multiples of the identity in the final $U(1)$. Since the local equivalence classes are orbits of this group, their dimension cannot be greater than the dimension of the group. Thus we have

Corollary 1. If $n \geq 3$, the generic local equivalence class for a system of $n$ $d$-state particles has dimension $n\left(d^{2}-1\right)+1$. 
Since a generic orbit has the same dimension as the group, the stabiliser of any state in the orbit has dimension zero. This gives

Corollary 2. The generic pure state of a system of more than two equal-spin particles has a discrete stabiliser under the action of local unitary transformations.

The stabilisers of states of three qubits $\left(d_{1}=d_{2}=d_{3}=2\right)$ were studied in [4]. The second corollary generalises Theorem 1 of that paper. This treatment, however, gives no indication of which exceptional states have enlarged stabilisers. This question is being investigated in an alternative approach by one of us (HAC).

For the general situation we must distinguish between the cases $d_{n} \leq D$ and $d_{n}>D$ where $D=d_{1} \ldots d_{n-1}$. In both cases, the number of zero coefficients imposed by conditions $1-2$ of Theorem 2 is

$$
\begin{aligned}
& \sum_{i=1}^{d_{1}-1} \sum_{s=1}^{n}\left(d_{s}-i\right)+\sum_{r=1}^{n-2} \sum_{i=d_{r}}^{d_{r+1}-1} \sum_{s=r+1}^{n}\left(d_{s}-i\right) \\
= & \left(d_{1}-1\right) \sum_{s=1}^{n} d_{s}+\sum_{r=1}^{n-2}\left(d_{r+1}-d_{r}\right) \sum_{s=r+1}^{n} d_{s}-n S_{1}-\sum_{r=1}^{n-2}(n-r)\left(S_{r+1}-S_{r}\right) \\
= & -\sum_{s=1}^{n} d_{s}+\sum_{r=1}^{n-1} d_{r}^{2}-\sum_{r=1}^{n-1} S_{r}-S_{n-1}+d_{n} d_{n-1} \quad \frac{1}{2} d_{r}\left(d_{r}-1\right) \\
= & \frac{1}{2} \sum_{r=1}^{n} d_{r}\left(d_{r}-1\right)-\frac{1}{2} \delta(\delta+1) \quad \text { where } \delta=d_{n}-d_{n-1} .
\end{aligned}
$$

If $d_{n} \leq D$, the number of zero coefficients imposed by condition 3 is $\frac{1}{2} \delta(\delta+1)$, while the number of phases removed by condition 4 is

$$
\sum_{r=1}^{n}\left(d_{r}-1\right)+1
$$

Hence the number of real parameters in the above canonical form is

$$
2 \prod_{r=1}^{n} d_{r}-\left(\sum_{r=1}^{n}\left(d_{r}^{2}-1\right)+1\right)
$$

which is the difference between the dimension of the state space and the dimension of the group $G$ of local transformations. Thus in this case the 
dimension of the generic local equivalence class is the same as the dimension of $G$, as in Corollary 1 .

If $d_{n}>D$, the number of zero coefficients imposed by condition 3 is

$$
\sum_{k=1}^{N}(\delta-k+1)=N(\delta+1)-\frac{1}{2} N(N+1)
$$

giving a total number of zero coefficients determined by conditions 1-3 as

$$
\begin{aligned}
& \frac{1}{2} \sum_{r=1}^{n} d_{r}\left(d_{r}-1\right)-\frac{1}{2} \delta(\delta+1)+\frac{1}{2} N(2 \delta-N+1) \\
= & \frac{1}{2} \sum_{r=1}^{n} d_{r}\left(d_{r}-1\right)-\frac{1}{2} \Delta(\Delta-1)
\end{aligned}
$$

where $\Delta=\delta-N+1=d_{n}-D$. In this case there are no non-zero coefficients $c_{i_{1} \cdots i_{n}}$ with $i_{n}>D$, so the number of phases removed by condition 4 is reduced by $\Delta$. Hence the total number of parameters removed, i.e. the dimension of the orbit, is at least

$$
\sum_{r=1}^{n}\left(d_{r}^{2}-1\right)+1-\Delta^{2}=\operatorname{dim} G-\Delta^{2}
$$

where $G=S U\left(d_{1}\right) \times \cdots \times S U\left(d_{n}\right) \times U(1)$.

The fact that there are no non-zero coefficients $c_{i_{1} \cdots i_{n}}$ with $i_{n}>D$ means that the state is unaffected by unitary transformations of the $n$th particle which fix the first $D$ basis vectors. Thus the stability group of the state contains at least this $U(\Delta)$ subgroup, and the dimension of the orbit cannot be greater than $\operatorname{dim} G-\Delta^{2}$. It follows that the dimension of the orbit is exactly this, and the Lie algebra of the stability group is exactly that of $U(\Delta)$

Thus the general versions of Corollaries 1 and 2 are

Corollary 3. In the general n-party system of Theorem 2, where $n \geq 3$, the generic orbit has dimension

$$
\begin{gathered}
\sum_{r=1}^{n}\left(d_{r}^{2}-1\right)+1 \quad \text { if } \quad d_{n} \leq D, \\
\sum_{r=1}^{n}\left(d_{r}^{2}-1\right)+1-\left(d_{n}-D\right)^{2} \quad \text { if } \quad d_{n}>D,
\end{gathered}
$$

where $D=d_{1} \cdots d_{n-1}$. 
Corollary 4. In the n-party system of Theorem 2, a generic point has discrete stabiliser in the group of local unitary transformations if $d_{n} \leq D$; otherwise the stabiliser is locally isomorphic to the unitary group $U\left(d_{n}-D\right)$.

\section{Alternative generalisations of the Schmidt decomposition}

The canonical form of the previous section is noteworthy for the simplicity of the conditions on the coefficients in the expansion of the state vector in terms of a factorisable orthonormal basis: certain coefficients are zero, certain others are real. This is likely to make it most useful in practice. However, it is perhaps less theoretically appealing than some other generalisations of the Schmidt decomposition that have been proposed recently. For the sake of completeness, we review these alternatives here.

For each constituent of the multipartite system, a basis of its individual state space is determined by its marginal density matrix: this is the basis defined by the conventional Schmidt decomposition of the state vector when the multipartite state space is regarded as a bipartite tensor product, one factor being the state space of the constituent being considered, the other being the tensor product of all the other state spaces. One of us [11] and Brun and Cohen [2] have proposed that the tensor products of these oneparticle states provide a natural basis for multipartite states. The resulting coefficients $c_{i_{1} \cdots i_{n}}$ satisfy

$$
\sum_{i_{1} \ldots i_{r-1}, i_{r+1} \ldots i_{n}} c_{i_{1} \cdots i_{r} \cdots i_{n}} \overline{\overline{i_{1} \cdots i_{r-1} j_{r} i_{r+1} \cdots i_{n}}}=0 \quad \text { if } \quad i_{r} \neq j_{r}
$$

for each $r$.

Spekkens and Sipe [10 have suggested that a canonical state in each equivalence class could be taken to be that which minimises the IngardenUrbanik entropy

$$
S_{\mathrm{IU}}=-\sum_{i_{1} \cdots i_{n}}\left|c_{i_{1} \cdots i_{n}}\right|^{2} \log \left|c_{i_{1} \cdots i_{n}}\right|^{2} .
$$

They justify this as a generalisation of the Schmidt decomposition by showing that the IU entropy is minimised by the Schmidt normal form for bipartite states. For more than two constituent parts, however, little is known about these minima.

To show that all three of these canonical forms are distinct, consider the tripartite state

$$
|\Psi\rangle=\frac{1}{2 \sqrt{3}}(3|000\rangle+|011\rangle+\sqrt{2}|111\rangle)
$$


where each constituent system is a qubit (a two-state system) and, as usual, we label the basis states by the digits 0 and 1 and abbreviate the product basis states as $|a b c\rangle=\left|\psi_{a}^{(1)}\right\rangle\left|\psi_{b}^{(2)}\right\rangle\left|\psi_{c}^{(3)}\right\rangle$. This state is presented in the canonical form of section 2; not only does it satisfy the conditions of Theorem 1, but it can be shown (see Appendix) that it is obtained by the procedure of that theorem, i.e. the coefficient of $|000\rangle$ is maximal among states locally equivalent to $|\Psi\rangle$. (We note that there is a locally equivalent state with the coefficient of $|000\rangle$ equal to $\frac{1}{2}$; this satisfies conditions 1 and 2 of Theorem 1 , but not condition 3.) By means of a transformation of the first qubit with matrix $\left.\frac{1}{\sqrt{6}}\left(\begin{array}{cc}\sqrt{2}+1 & 1-\sqrt{2} \\ \sqrt{2}-1 & \sqrt{2}+1\end{array}\right)\right)$, the state $|\Psi\rangle$ is locally equivalent to

$$
|\Phi\rangle=\frac{1}{2 \sqrt{2}}((\sqrt{2}+1)|000\rangle-(\sqrt{2}-1)|100\rangle+|011\rangle+|111\rangle)
$$

which is in the canonical form of [1] and [2]. Neither of these states minimises the IU entropy within their local equivalence class: under the infinitesimal local transformation in which the basis states of the first qubit transform by $|0\rangle \mapsto|0\rangle+\epsilon|1\rangle,|1\rangle \mapsto|1\rangle-\epsilon|0\rangle$ and the states of the second and third qubits are kept fixed, the entropies of $|\Psi\rangle$ and $|\Phi\rangle$ change by

$$
\delta S_{\mathrm{IU}}(\Psi)=-\frac{1}{3 \sqrt{2}} \epsilon \log 2, \quad \delta S_{\mathrm{IU}}(\Phi)=-\epsilon \log (\sqrt{2}+1) .
$$

By minimising the IU entropy numerically we find in general that a few of the coefficients $c_{i j k}$ become much smaller than the others without vanishing exactly.

\section{Acknowledgement}

We are grateful to Bob Gingrich for pointing out an error in an earlier version of this paper. 


\section{Appendix}

We will show that for a state

$$
|\Psi\rangle=a|000\rangle+b|011\rangle+c|111\rangle
$$

with $|a|>\frac{1}{\sqrt{2}}, a$ is the maximal value of the coefficient of $|000\rangle$ among states locally equivalent to $|\Psi\rangle$.

Proof. The equations (2.9) for a stationary value of $\left|\left\langle\Psi\left|\left(\left|\phi^{(1)}\right\rangle\left|\phi^{(2)}\right\rangle\left|\phi^{(3)}\right\rangle\right)\right|^{2}\right.\right.$ become

$$
\begin{aligned}
\bar{a} v_{0} w_{0}+\bar{b} v_{1} w_{1} & =\lambda \overline{u_{0}} \\
\bar{c} v_{1} w_{1} & =\lambda \overline{u_{1}} \\
\bar{a} u_{0} w_{0} & =\lambda \overline{v_{0}} \\
\bar{b} u_{0} w_{1}+\bar{c} u_{1} w_{1} & =\lambda \overline{v_{1}} \\
\bar{a} u_{0} v_{0} & =\lambda \overline{w_{0}} \\
\bar{b} u_{0} v_{1}+\bar{c} u_{1} v_{1} & =\lambda \overline{w_{1}}
\end{aligned}
$$

where $\left|\phi^{(1)}\right\rangle=u_{0}|0\rangle+u_{1}|1\rangle,\left|\phi^{(2)}\right\rangle=v_{0}|0\rangle+v_{1}|1\rangle$ and $\left|\phi^{(3)}\right\rangle=w_{0}|0\rangle+w_{1}|1\rangle$. Clearly there is a solution $\lambda=\bar{a}, u=v=w=(1,0)$. We have to show that any other solution has $|\lambda|^{2}<|a|^{2}$. Using (3.5) and (3.6) to eliminate $w_{0}$ and $w_{1}$, eqs. (3.1) and (3.2) become

$$
\begin{array}{r}
\left(|a|^{2}\left|v_{0}\right|^{2}+|b|^{2}\left|v_{1}\right|^{2}-|\lambda|^{2}\right) u_{0}+b \bar{c}\left|v_{1}\right|^{2} u_{1}=0 \\
\bar{b} c\left|v_{1}\right|^{2} u_{0}+\left(|c|^{2}\left|v_{1}\right|^{2}-|\lambda|^{2}\right) u_{1}=0 .
\end{array}
$$

For $\left(u_{o}, u_{1}\right) \neq(0,0)$, it follows that

$$
F\left(|\lambda|^{2}\right)=|\lambda|^{4}-|\lambda|^{2}\left(|a|^{2}+\left(1-2|a|^{2}\right)\left|v_{1}\right|^{2}\right)+|a|^{2}|c|^{2}\left|v_{0}\right|^{2}\left|v_{1}\right|^{2}=0
$$

since $|a|^{2}+|b|^{2}+|c|^{2}=\left|v_{0}\right|^{2}+\left|v_{1}\right|^{2}=1$. Now

$$
F\left(|a|^{2}\right)=|a|^{2}\left|v_{1}\right|^{2}\left(2|a|^{2}-1+|c|^{2}\left|v_{0}\right|^{2}\right) \text {. }
$$

which is positive if $|a|>\frac{1}{\sqrt{2}}$ (unless $v_{1}=0$ ), and the gradient of the quadratic $F$ at $|\lambda|^{2}=|a|^{2}$ is also positive. It follows that the zeros of $F$, and therefore any stationary values of $\left|\left\langle\Psi\left|\left(\left|\phi^{(1)}\right\rangle\left|\phi^{(2)}\right\rangle\left|\phi^{(3)}\right\rangle\right)\right|^{2}\right.\right.$ other than $|a|^{2}$, are less than $|a|^{2}$. 


\section{References}

[1] A. Acín, A. Andrianov, L. Costa, E. Jané, J. I. Latorre, and R. Tarrach, Schmidt decomposition and classification of three-quantum-bit states, quant-ph/0003050.

[2] T. A. Brun and O. Cohen, Parametrization and distillability of threequbit entanglement, quant-ph/0005124.

[3] H. A. Carteret, N. Linden, S. Popescu, and A. Sudbery, Multi-particle entanglement, Found. Phys. 29 (1999), 527.

[4] H. A. Carteret and A. Sudbery, Local symmetry properties of pure states of three qubits, J. Phys. A 33 (2000), 1-22, quant-ph/0001091.

[5] V. Coffman, J. Kundu, and W. K. Wootters, Distributed entanglement, Phys. Rev. A 61 (2000), 2306, quant-ph/9907047.

[6] M. Grassl, M. Rötteler, and T. Beth, Computing local invariants of qubit systems, Phys. Rev. A 58 (1998), 1853, quant-ph/9712040.

[7] J. Kempe, Multi-particle entanglement and its applications to cryptography, Phys. Rev. A 60 (1999), 910, quant-ph/9902036.

[8] N. Linden and S. Popescu, On multi-particle entanglement, Fortschr. Phys. 46 (1998), 567, quant-ph/9711016.

[9] Y. Makhlin, Nonlocal properties of two-qubit gates and mixed states and optimization of quantum computations, quant-ph/0002045.

[10] R. W. Spekkens and J. E. Sipe, Non-orthogonal preferred projectors for modal interpretations of quantum mechanics, quant-ph/3092.

[11] A. Sudbery, On local invariants of pure three-qubit states, quantph/0001116.

[12] A. V. Thapliyal, On multipartite pure state entanglement, Phys. Rev. A 59 (1999), 3336, quant-ph/9811091.

[13] W. K. Wootters, Quantum entanglement as a quantifiable resource, Phil. Trans. Roy. Soc. A 356 (1998), 1717. 